\title{
Drought Monitoring Based on the Vegetation Temperature Condition Index by IDL Language Processing Method
}

\author{
WenHao Ou, Wei Su*, Chen Wu, ZhongZheng Zhu, YanMin Li, and Shi Shen \\ College of Information and Electrical Engineering, \\ China Agricultural University, Beijing, 100083 \\ suwei@cau .edu.cn
}

\begin{abstract}
Landsat TM5 images are used to calculate and retrieve normalized difference vegetation index (NDVI) and land surface temperature (LST). Combining with two index mentioned, vegetation temperature condition index (VTCI) can be retrieved for drought monitoring indicator applied in Junchuan farm of Heilongjiang Province in Northeast China. With well performance in matrix operation of IDL language, retrieving VTCI in a short time, fast batch calculation and mapping work as well, to a great extent, saving time and laborites, also providing real-time data for the government's macroeconomic regulatory policy.
\end{abstract}

Keywords: VTCI, Drought Monitoring, Batch Processing, IDL.

\section{Introduction}

Drought, which happens periodically with complex procedure, has been a kind of natural catastrophe that mankind has to deal with of all time. It takes place in all kinds of climate zones and is listed as one of the main natural catastrophes around the world. Of all kinds of natural catastrophes, drought has the strongest impact on agriculture. According to conservative statistics, drought takes $61 \%$ percent of the disaster-affected area in China, while flood takes $24 \%$, hail takes $9 \%$, and frost takes $6 \%[1-2]$. Traditional method to monitor a drought is by using meteorological data to decide the level and range and it is only restrict to monitoring on a certain spot. However, in order to monitor a large area of drought condition, remote sensing should be used as the main method [3-4].

With the development of remote sensing techniques, available data have been increasing all the time. To monitor drought, vegetation index (NDVI) and land surface temperature (LST) are often used to retrieve. NDVI is to express the condition of vegetation by using the spectral character of red light, near infrared band or their simple combination or linear combination, based on the strong absorbing property that chlorophyll has on lights [5]. There are many indexes to show the drought condition, for example, Anomaly Vegetation Index (AVI), Vegetation Condition Index (VCI), Land Surface Temperature (LST), Vegetation Temperature Condition Index (VTC) etc.

* Corresponding author. 
VTCI is based on the triangle composed of scatter diagram (feature space) of Normalized Difference Vegetation Index (NDVI) and LST and is used to study the drought level and its variance of a certain area during a certain period of a year. It takes the variance of NDVI into consideration and at the same time, the variance of LST under the same NDVI, that is, the harm that high temperature can have on plants [6]. This method, which is put forward by Pengxing Wang, is now used to monitor the drought in Shanxi Province (China), Texas and Oklahoma (USA). Results show that it is an effective way and can be a real-time procession [7].

In order to provide data faster and better, VTCI by IDL language processing method, combined with remote sensing image, should be used. IDL is a simple matrix-oriented and cross-platform language and it has the ability to process a large amount of data in high speed. Therefore, IDL language is an ideal tool to process remote sensing image [8]. This paper is mainly discussing drought monitoring based on the vegetation temperature condition index by IDL language processing method.

\section{Basic Information about Research Area and Data Resource}

In 2007, Heilongjiang Province suffered from the most serious drought in 50 years and then the importance of drought monitor emerged. Take the Junchuan farm (115/027 in TM5) for example. It is a temperate continental climate place located at $131^{\circ} 02^{\prime}$ to 131 ${ }^{\circ} 30^{\prime}$ east longitude, $47^{\circ} 20^{\prime}$ to $47^{\circ} 40^{\prime}$ northern latitude in Luobei and Suibin. We analyse the drought condition on the farm to capture the level of the drought. This paper use VTCI, combined with LandSat TM5 remote sensing data to monitor the drought, providing the support for some decisions on the farm.

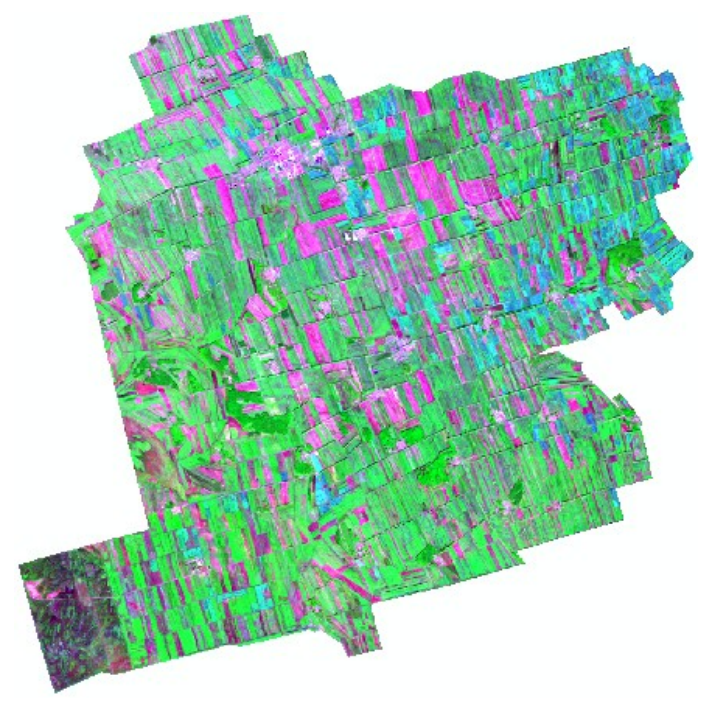

Fig. 1. Landsat TM5 image of study area (Red: Band7, Green: Band4, Blue: Band3)

In the process of pre-processing original $\mathrm{TM}$ remote sensing image, radiative correction and geometrical correction are usually carried out. Radiative correction is 
the first setp, followed by atmospheric correction. Then geometrical correction is carried out to pick some control point on the image by polynomial method. Gauss-Kruger projection is used to do the geometrical correction. All the steps above is processed by ENVI 4.7.

\section{Results and Analysis of Drought Monitor by IDL}

\subsection{Extraction of VTCI}

1) Normalized vegetation index is calculated by Landsat TM5, band 3 and band 4 . The formula is as below:

$$
N D V I=\frac{\rho_{4}-\rho_{3}}{\rho_{4}+\rho_{3}}
$$

$\rho_{4}$ refers to reflectivity of band 4 , and $\rho_{3}$ refers to that of band 3 .

2) The calculation of land surface bright temperature

$$
B T=\frac{K_{2}}{\ln \left(\frac{K_{1}}{L_{\lambda}}+1\right)}
$$

$L_{\lambda}$ refers to radiance of thermal infrared band. $K_{1}$ and $K_{2}$ are calibration constants. $K_{1}$ equals 607.76 and $K_{2}$ equals to 1260.56 because of the empirical model of Landsat-5 data.

3) The calculation of land surface temperature

Land surface temperature is calculated by Landsat TM5, band 6, mainly the surface thermal infrared radiation [9].

$$
L S T=\frac{B T}{\varepsilon^{1 / 4}}
$$

$\mathcal{E}=1.009+0.047 \ln N D V I$, BT refers to the spectrum radioactive brightness.

4) The calculation of VTCI

$$
V T C I=\frac{L S T_{N D V I_{i} \max }-L S T_{N D V I_{i}}}{L S T_{N D V I_{i} \max }-L S T_{N D V I_{i} \min }}
$$

(Formula 4)

$L S T_{N D V I_{i}}=a+b^{*} N D V I ; L S T_{N D V I_{i} \min }=a^{\prime}+b^{\prime *} N D V I$. $a, b, a^{\prime}, b^{\prime}$ are undetermined coefficient and need to be determined by linear fitting on borderline of scatter diagram. 


\subsection{Export VTCI Image with ENVI/IDL}

According to requirements above, we designed a reasonable scheme, which can be processed spontaneously.

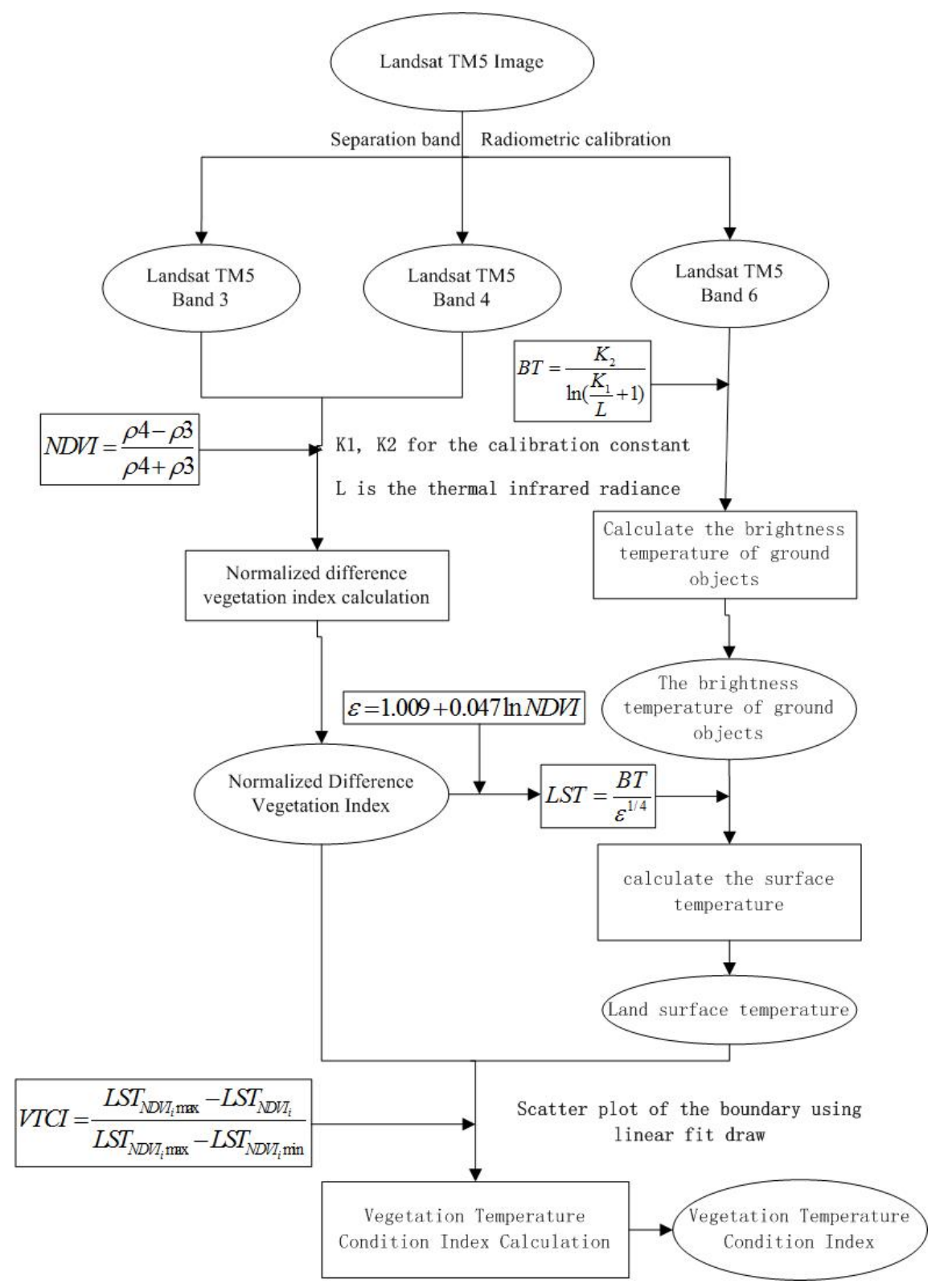

Fig. 2. Computing in the process of IDL 
According to figure 2, firstly, wave band separation and radioactive calibration are carried out on TM5 image. Secondly, extracted band 3, band 4 and band 6 are calculated in NDVI, BT AND LST. At last, calculate VTCI and output VTCI image by fitting hot and cold borderline.

1) TM5 Band3 radiation calibration calculation method (DegreeToRad for custom Angle turn radian function name):

function compute_l, origin

result $=$ float $($ origin $) * 1.039880-1.17$

$\mathrm{e}=1554$; According to the atmosphere of solar spectrum top average irradiance $\mathrm{d}=1 ; \mathrm{d}$ to the distance for the day result $=$ !pi $* 1 * \mathrm{~d} * \mathrm{~d} / \mathrm{e} / \cos (\operatorname{DegreeToRad}(25))$; For radiation correction return, result

end

2) According to TM5 band 3 and band 4 the calculation method of NDVI (according to the above Formula 1) :

function compute_ndvi, b1, b2, check $=$ check

den $=$ float $(\mathrm{b} 1)+$ float $(\mathrm{b} 2)$

if $($ keyword_set $($ check $))$ then $\operatorname{ptr}=$ where $($ den eq 0 ., count $) \$$

else count $=0$

if ( count gt 0 ) then den[ptr] $=1.0$

result $=($ float $(\mathrm{b} 1)-$ float(b2) $) /$ den $+0.00001 ;$ In order to avoid value, plus 0.00001

if (count gt 0 ) then result $[\mathrm{ptr}]=0.0$

return, (float(b1)-float(b2))/(float(b1)+float(b2)+0.001)

end

3) Inversion of LST (Formula 3):

function compute_lst, bt, ndvi

result $=$ fltarr(n_elements $($ ndvi $[*, 0])$, n_elements $\left(\right.$ ndvi $\left.\left.\left[0,{ }^{*}\right]\right)\right)$

$\mathrm{e}=1.009+0.047 * \operatorname{alog}(\mathrm{ndvi}) ;$ Calculate e

result $=$ float $(b t]) / \mathrm{e}^{\wedge}(0.25)$

return, result

end 
4) Calculation VTCI according to the results of NDVI and LST:

function compute_vtci, ndvi, lst, aw, bw, ac, bc
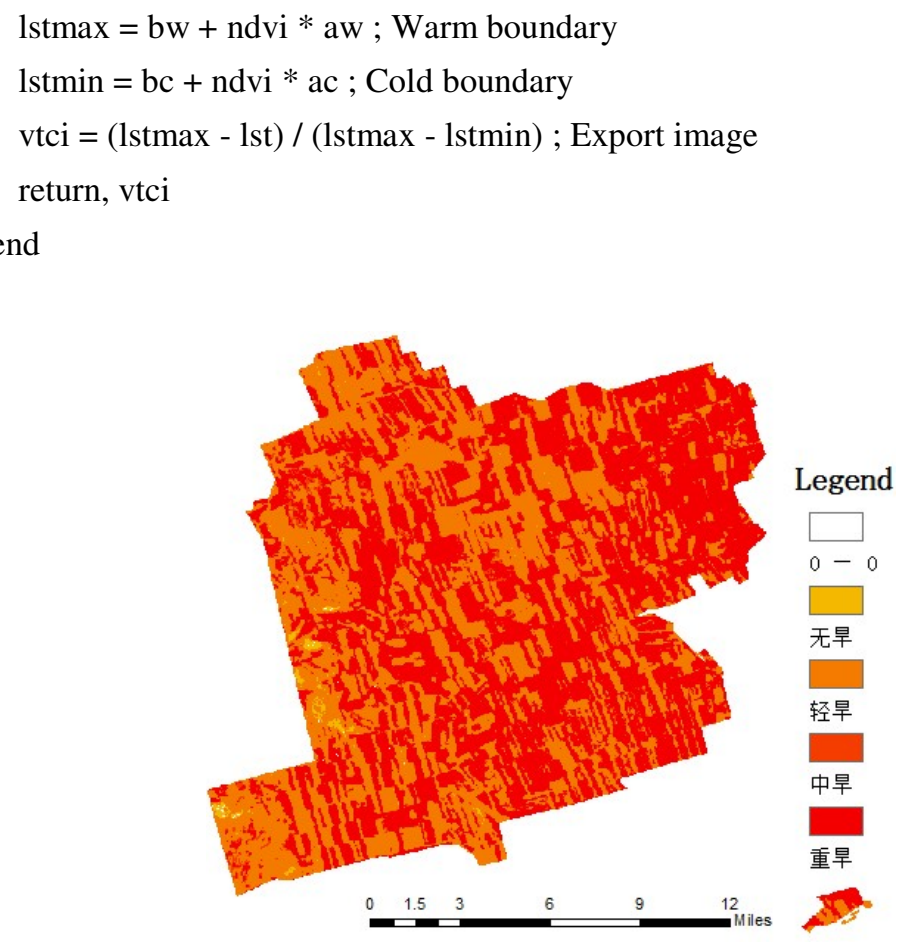

Fig. 3. Result of VTCI monitored in JunChuan farm

In the program above, ";" is a sign of comment. We can see that IDL is a language of simple grammar. It processes the matrix as a whole without pixels by pixels and it saves a lot of time when processing large matrixes. By using the method above, it took 18.658 seconds to process this image of Junchuan farm on September 25th, which saves much time than hand operation, and there's no need to wait in front of the computer for the next step. Figure 3 is the resulting VTCI image. From it we can see that the area is in middling drought or severe drought, which is exactly the same with the real condition.

\section{Conclusion}

This paper uses spontaneous and time-saving method to retrieve VTCI based on IDL language as an example to put forward a way to process large amount of remote sensing data also spontaneously and automatically to monitor drought condition. By using IDL, we can realize that:

1) Modularization of the process and the ability to be transplanted through different operating systems. 
2) All-day automatic work, which can output real-time VTCI result, and make it easier for government to know the drought and take emergency measurement.

3) Once successfully set, there's no need to interfere or guard it with labor, which is time and labor saving. Computers can make automatic procession and save result as the settings, file names and path, which can avoid mistakes.

Acknowledgments. This research is funded by the Natural Science Foundation of China entitled Estimation of tree height using LIDAR data and QuickBird imagery (40801128). All of the authors appreciate the co-operation and support of many experts from the Research Institute of Forest Resource Information Techniques of Chinese Academy of Forestry, and we all would like to thank Professor Liu Qinfu of the College of Geoscience and Surveying Engineering, China University of Mining \& Technology (Beijing), for his support with human resources.

\section{References}

[1] Wang, P., Wan, Z., Gong, J., et al.: Drought monitoring mode based on vegetation index and land surface temperature. Earth Science Progress 8(18), 527-533 (2003)

[2] Wang, X., Guo, N.: Progress of drought monitoring and the research methods of remote sensing. Arid Meteorological, 77-80 (2003)

[3] Lambin, E.F., Ehrlich, D.: The surface temperature-vegetation index for land cover and land cover change analysis. International Journal of Remote Sensing 17, 463-487 (1996)

[4] Liu, W., Kogan, F.N.: Monitoring regional drought using the vegetation condition index. International Journal of Remote Sensing 17, 2761-2782 (1996)

[5] Chen, S., Tong, Q., Guo, H.: Reaserch of remote sensing information. Beijing Science Press (1998)

[6] Wang, P., Gong, J., Li, X.: The application of drought monitoring with vegetation temperature condition index. Journal of WuHan University, Information Science Edition 10(26), 412-418 (2001)

[7] Wan, Z., Wang, P., Li, X.: Using MODIS land surface temperatureand normalized difference vegetation index products for monitoring drought in the southern Great Plains, USA. International Journal of Remote Sensing 25(1), 61-72 (2004)

[8] Quan, T., Zheng, N.: Remote sensing systems development methods based on the secondary devel-opment with ENVI/IDL. Computer Application 6(28), 270-276 (2008)

[9] Liu, Z., Lei, Z., Dang, A., et al.: Remote sensing technology and SEBAL model in the application of arid evaporation estimation. Journal of Tsinghua University (Natural Science Edition) 44(3), 279-679 (2004) 